Among HIV-Positive Adults With Different Sexual Identities. Behavioral Medicine, 34(4), 133-144

NENGEH. M. (2003). Ni vues ni connues? Femmes, VIH, Médias. Montréal: Les éditions du remue-ménage.

ONUSIDA (2009). Le point sur l'épidémie du sida décembre. [en línea] http://data.unaids.org/pub/ Report/2009/jc1700_epi_update_2009_fr.pdf, consultado 15 de enero 2010.

OSTROM, A., M. SEROVICH, J. LIM \& T. MASON (2006). The role of stigma in reasons for HIV disclosure and non-disclosure to children, AIDS Care, 18(1) 60-65.

PÁEZ, D., ECHEbarRía, A., VALENCiA, J., ROMO I., SAN JUAN, C., \& VERGARA, A. (1991). AIDS social representations: Contents and processes. Journal of Community and Applied Social Psychology, 1, 89-104.

PETERSON, S., C. MARTINS \& J. COFRANCESCO (2008). Lipodystrophy in the patient with HIV: social, psychological, and treatment consid

PRESTON, D.B., E.M. FORTI, C. KASAD \&, P.B. KOCH (2000). Personal and social determinants of rural nurses' willingness to care for persons with AIDS. Research in Nursing Health, 23, 67-68.

QUINN, T. (2008). HIV epidemiology and the effects of antiviral therapy on long-term consequences. AIDS 22(3), S7-12

RAMÍREZ-VALLES J., S. FERGUS, C. REINSEN, P. POPPEN \& M-C ZEA (2005). Confronting Stigma Community Involvement and Psychological WellBeing Among HIV-Positive Latino Gay Men. Hispanic Journal of Behavioral Sciences, 27(1), 101-119.

ROBERTS, J.K. (2002). Physician-Patient Relationships, Patient Satisfaction, and Antiretroviral Medication Adherence Among HIV-Infected Adults Attending a Public Health Clinic. AIDS Patient Care and STDs, 16(1), 43-50

STRONG, P. (1990). Epidemic psychology: a model. Sociology of Health \& Illness, 12(3), 249-259.

STRAUSS, A. \& J. CORBIN (1990). Basic of Qualitative Research. Grounded theory Procedures and Techniques, Newbury Park California: Sage Publication.

SULLIVAN, L.M., J.B. SAVETSKY \& J.H. SAMET (2000). The doctor-patient relationship and HIV-infected patients' satisfaction with primary care physicians. Journal of General Internal Medicine, 15(7), 462-469.

TEIXEIRA M.G. \& G.A. SILVA (2008). Representation of he HIV carriers about antiretroviral treatment. Revis da Escola de Enfermagem da USP. 42(4), 729-736.

THOMAS, F. (2006). Stigma, fatigue and social breakdown: Exploring the impacts of HIV/AIDS on patien and carer well-being in the Caprivi Region, Namibia. Social Science \& Medicine 639(12) 3.174-3 187.
VARAS-DÍAZ, N. \& M. MARZÁN-RODRÍGUEZ (2007). The emotional aspect of AIDS stigma among health professionals in Puerto Rico. AIDS Care, 19(10), 1.2471.257

VELIMIROVIC B. (1987) AIDS as a social phenomenon Social Science \& Medicine. 25(6), 541-552.

WALTER, F., (1988). AIDS 1988: The science and the limits of science. American Psychologist. 43(11), 853858

WEEKS, J. (1989). AIDS : The intellectual agenda, in AIDS : Social representation, Social Practices, Lewes, East Sussex, England : Falmer Press.

WELLS, B. (2009). The impact of mental health and traumatic life experiences on antiretroviral treatment outcomes for people living with Hiv/

ZELAYA, C., S. SIVARAM, S. C. JOHNSON, A. K. SRIKRISHNAN, S. SOLOMON \& D. CELENTANO (2008). HIV/AIDS Stigma: Reliability and Validity of a New Measurement Instrument in Chennai, India. AIDS and Behaviors, 12(5), 781-788.

\section{Repensando la política de salud y pueblos indígenas a partir de la experiencia en el CESFAM Rodelillo ¿qué es lo intercultural de la interculturalidad en salud?}

Re-thinking health policy and indigenous people from CESFAM Rodelillo's experience: What is the intercultural of the intercultural health?

Mario Catalán Catalán

Trabajador Social Pontificia Universidad Católica de Valparaíso, Chile.

- Programa de Intervención Breve Villa Alemana Corporación SERPAJ-Chile. (Desde Abril 2012 a la fecha) - Centro de Educacion en Ecologia y Desarrollo Humano JUBAEA.

Correo electrónico: mariocatalan.c@gmail.com Dirección postal: Calle Yungay \#2549, Depto. 144, Valparaíso, Chile

\section{Resumen}

El desafio es repensar lo que se entiende por salud intercultural en Chile; quizás este término ha sido sobreutilizado por la política de salud para referirse a estrategias de integración de la cultura del usuario al proceso de atención, las cuales se orientan a relaciones interétnicas entre sujetos con distinta pertenencia cultural y no a relaciones interculturales, que consisten en la interacción, relación, negociación, diálogo ylo préstamos recíprocos de sujetos con mallas de significado diversas. A partir de la interpretación de significados construidos por sujetos implicados en la avención Ae medicina interpretación de significados construidos por sujetos implicados en la pregunta: ise ha cuestionado lo litica de salud sobre la influencia en la práctica médica mapuche que, ha custionado la politica de salud sobre la influencia en la práctica médica maescritorio, en el momento de la atención, el machi y el usuario se encuentren separados por un propio de dicha práctica ancestral?

Palabras clave. Interculturalidad en salud, cultura mapuche, politica de salud y pueblos indígenas significados.

\section{Abstract}

The challenge is to re-think what is understood by intercultural health in Chile: perhaps the term has been overused by health policy to refer to integration strategies of the user's culture to the process of care, which are oriented to interethnic relations among subjects with different cultura belonging, and not to intercultural relations, which consist in interaction, relationship, negotiation, dialogue and / or reciprocal borrowings of subjects with diverse meaning meshes. From the interpretation of meanings constructed by individuals involved in the Mapuche medical attention in a particular situation, this article invites to reflect on the following question: has health policy questioned itself about its influence in the medical Mapuche practice and in the fact that, at the time of care, machi and the user are separated by a desk or that the attention lasts fifteen minutes considering that this is not part of this ancestral practice?

Key words. Intercultural health, Mapuche culture, health policy and indigenous people, meanings. 


\section{Introducción}

\section{Contextualización atención de machi en el}

Centro de Salud Familiar Rodelillo, Ciudad de Valparaíso

Este artículo se construye en base a una investigación cualitativa de tipo comprensivo-interpretativa realizada entre marzo del 2011 y enero del 2012. El objetivo de la investigación estuvo orientado a interpretar los significados otorgados por los sujetos implicados al proceso de atención de medicin mapuche en el CESFAM Rodelillo (Centro de Salud Familiar de Rodelillo). La investigación se realiza en a Comunidad Mapuche Lof Cheuque - Asociació Indígena Rayen Foye, Cerro Rodelillo, Valparaíso Ella recibe por nombre: "Interculturalidad en Salud: Significados otorgados por sujetos implicados en el proceso de atención de medicina mapuche en el Cesfam

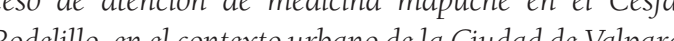
Rod. Dill en elcontextoubano de la Ciudad de Valparao . Dicha investigación tiene como intencionalidad, desde el Trabajo Social, ser un insumo para la gestión de la política de salud y la ejecución del program particular, al colocar de manifiesto los significado construidos por los sujetos a quienes va dirigida la acción estatal, rescatando las mallas de significación singular en la construcción de conocimiento crítico.

La Política de Salud y Pueblos Indígenas tiene como propósito "contribuir al mejoramiento de la situación de salud de los pueblos originarios, a través del desarrollo progresivo de un modelo de salud con enfoque intercultural" (MINSAL, 2003), y propone integrar en el espacio de los Centros de Salud, prácticas propias de los pueblos originarios relacionadas con uración de enfermedades. Para este propósito, se plantea el Programa Especial de Salud y Pueblos Indígenas (PESPI), dependiente de a Subsecretaría de Redes Asistenciales del Minsa (Ministerio de Salud), el cual tiene como propósito que las poblaciones indígenas de las zonas urbanas y rurales mejoren su condición de salud (MINSAL 2011), esto a través de la participación de las comunidades en búsqueda de la equidad en tanto al acceso a la salud, para lo cual propone que las mismas comunidades (agrupaciones o asociaciones) ndígenas elaboren sus propios proyectos, los cuales son postulados a recursos que entrega este programa. Estos proyectos abarcan tanto desde la atención de machi, celebración de ritos tradicionales (We Tr Pantü, Ngüillatún, etc.), y la difusión de actividades y talleres (de cosmovisión mapuche, yerbas medicinales, etc.).
En la ciudad de Valparaíso este tipo de intervención comenzó en el año 2001 a cargo del Longko de la Comunidad Mapuche Urbana We Folilche Amuleaiñ en cerro Cordillera. Un año más tarde se suma la Asociación Indígena Rayen Foye.

Esta asociación realiza su intervención en tres Centros de Salud de la ciudad de Valparaíso (Rodelillo, Barón y Placeres), una vez al año en cada centro, los cuales tienen características urbanas. Según estadísticas de la Asociación, se atienden alrededor de 30 personas en cada CESFAM, de las cuales un $90 \%$ no tiene ascendencia étnica mapuche, mientras que el $10 \%$ si la tiene. Entre las enfermed des que predo minan en las consultas encontramos las siguientes: dolores de huesos, sistema nervioso, dolores musculares, diabetes, hipertensión, colesterol, sistema respiratorio, artrosis, colon irritable, entre otras ${ }^{1}$

La gestión y coordinación de la intervención de atención de machi es realizada por el Encargado Mapuche, el encargado del Servicio de Salud ValServicio de Salud Valparaíso San Antonio y el Director del respectivo Centro de Salud. La Asociación realiza un período de difusión de la atención que se realizará (aproximadamente 3 semanas antes de realizarla), y luego realiza la atención de machi propiamente tal durante una mañana, una vez al año.

La atención de machi en el Centro de Salud Rodelillo comienza alrededor de las 09:00 horas y finaliza aproximadamente a las 14:00 horas; es por orden de llegada y dura aproximadamente 15 minutos. El Centro de Salud facilita una sala de atención para que el machi pueda desarrollar su práctica curativa. El usuario entra a la sala de atención, el machi le realiza algunas consultas respecto del motivo de la atención y entrega yerbas medicinales, las cuales se encuentra selladas al interior de una bolsa de plástico, para la sanación de la enfermedad, la cual identifica a partir del relato de las dolencias del sujeto. El machi se ubica detrás de un escritorio desde donde interactúa con el usuario

\section{Construcción teórica-conceptual}

Ahora bien, respecto de la construcción teórica del estudio, es necesario señalar que este se elabora a partir del diálogo entre diversos conceptos que permiten comprender la realidad desde una posición interpretativa de los fenómenos socioculturales. Se entiende cultura según la concepción semiótica planteada por Clifford Geertz, (2003) como un entramado de significaciones. Lo cual se relaciona con el concepto de Interculturalidad desarrollado por Néstor García Canclini, (2004) quien lo define como "la confrontación y entrelazamiento, a lo que sucede cuando los grupos entran en relaciones e intercambios (...) implica que los diferentes son lo que son en relaciones de negociación, conflicto y prestamos recíprocos" (p. 14-15), es decir la interculturalidad puede ser entendida como la relación de las diversas mallas de significación construidas por los sujetos en un espacio social particular. El autor citado coloca el concepto de interculturalidad en oposición al de multiculturalidad. Bajo concepciones multiculturales (yuxtaposición de etnias o grupos en una ciudad o nación) se admite la diversidad cultural, subrayando su dit proponiendo políticas relativistas de respeto, que a menudo refuerzan la segregación. La multiculturalidad supone adaptación de lo heterogéneo.

El concepto de significado se entiende como lo ha definido Jerome Bruner, (1998) "en virtud de nuestra participación en la cultura, el significado se hace público y compartido. Nuestra forma de vida, adaptada culturalmente, depende de significados y conceptos compartidos, y depende también de formas de discurso compartidas que sirven para negociar las diferencias de significado e interpretación" (p. 29). Los significados serían producto de las relaciones de negociación, conflicto e interacción tanto al interior de la propia cultura como en la interacción con culturas diversas.

Considerando la temática de estudio, se entiende interculturalidad en salud (o salud intercultural) como "aquellos procesos socioculturales referidos al ámbito de la salud, los cuales tendrían dos dimensiones: por un lado las acciones estatales de integración de la cultura de los pueblos originarios en el proceso de atención en el contexto del modelo biopsicosocial; y por otro, a la relación de negociación, conflicto, intercambio, encuentro o desencuentro entre culturas diversas, no necesariamente referido a las relaciones interétnicas (sino que más bien referidas a relaciones interculturales), en la que intervendría la interacción de las diversas tramas de significación construidas por los sujetos a partir de su experiencia particular" (definición elaborada por el autor). Esto permite entender la interculturalidad en salud como estrategias estatales en relación a fenómenos socioculturales y viceversa.

Además de lo anterior, tiene como consideración la premisa de considerar a los sistemas médicos como sistemas culturales, siguiendo a Aldo Vidal (en: Citarella, 1995), por ser estos sistemas de símbolos interpretables, una malla de significados respecto dependiendo del contexto en el cual se sitúa, lo que a su vez se relaciona con lo planteado por Geertz y García Canclini.

\section{Metodología de la investigación}

Coherentemente con el planteamiento teórico, la investigación se construye a partir de una metodología cualitativa con un enfoque comprensivo-interpretativo de los fenómenos sociales. La perspectiva epistemológica desde la cual se posiciona el investigador es la hermenéutica cultural partir de la articulación de los planteamientos de Gadamer (1998) y Geertz (1994, 1996, 2003); esto permite abocarse en el estudio de las produccione culturales, "estudio de los textos sobre la cultura, má que el de la cultura como texto" (Arriarán, 2000).

Como estrategia de acercamiento a la realidad, se define realizar un estudio de caso en el espacio del CESFAM Rodelillo, por los siguientes criterios: (1) Fue en el primer Centro de Salud donde la Asociación Indígena Rayen Foye (Lof Cheuque) realizó su intervención con medicina mapuche (atención de machi); (2) La Asociación Indígena Rayen Foye (Lo Cheuque) se encuentra ubicada geográficamente en el territorio de este Centro de Salud; (3) A este Centro de Salud acude la población mapuche del sector de Rodelillo a atención; (4) Este CESFAM posibilita el acceso a sujetos tales como usuarios mapuche $y$ no mapuche, y encargados mapuche de gestionar la atención de medicina mapuche en este contexto. Para la selección de los sujetos se opto por la realización de un muestreo teórico (Strauss \& Corbin 2002), donde los criterios fueron:

\section{A. Criterios de homogeneidad:}

A.1. Relación con el CESFAM Rodelillo.

A.2. Conocimiento de la intervención realizada por la Asociación Indígena Rayen Foye.

A.3. Disponibilidad de participar en la investigación.

B. Criterios de heterogeneidad:

B.1. Posición del sujeto en el proceso de atención:

B.1.1. Usuario atención de machi (mínimo dos atenciones):

B.1.1.1. Usuario mapuche.

B.1.1.2. Usuario no mapuche.

B.1.1.2.1. Usuario no mapuch profesional del CESFAM Rodelillo.

B.1.1.2.2. Usuario no mapuche no profesional de CESFAM Rodelillo. 
B.1.2. Encargado de gestionar la intervención con medicina mapuche.

Es de esta manera que los sujetos participantes de esta investigación tuvieron cuatro, tres usuarios de atención con medicina mapuche, de los cuales uno tenía pertenen tenia pertenencie tica mapuche y dos no latenían (uno de estos tambienn era profesional del CESFAM, mientras que el otro solo era usuario del CESFAM) y un sujeto encargado de gestionar la intervención con medicina mapuche

La técnica de producción de la información corresponde a la entrevista en profundidad, porque permite acceder a situaciones a las cuales el inves(Taylor \& Bogdan, 1987). Se entiende esta técnica como un constructo comunicativo y una interacción socia entre entrevistado e entrevistador, siguiendo a Luis Enrique Alonso (2003)

El método de análisis corresponde al método semántico de análisis de contenido (Navarro \& Díaz, 1994), el cual permite latente que cognivas que instrumentalnente recurren a la comunicación que instrumentalmente recurren a la colos actos comunicar la interaccion que subyace a los actos comunicativos concretos y la superficie material del texto. Se utiliza un proceso estándar, donde se definen unidades de registro y unidades de contexto, luego se codifican (se adscriben las unidades de registro en las unidades de contexto), posteriormente se categorizan y luego se interpret. luego se interpretan dichas categorías.

\section{Resultados del estudio: significados} construidos por los sujetos

Para exponer los principales resultados del estudio se responderán las dos preguntas de investigación planteadas.

La primera pregunta es, ¿Cuáles son los significados que los sujetos implicados otorgan al proceso de atención a partir de su experiencia en el Cesfam Rodelillo, en el contexto urbano de la ciudad de Valparaíso?, a través de esta pregunta se pretende develar las significacic sujetos implicados. La forma en la cual cada un de los sujetos signifique el proceso de atención de medicina mapuche depende de su experiencia personal, su historia vital, y del contexto sociocultural y político donde se sitúe en relación a los procesos de interculturalidad en salud.

Encargado mapuche de gestionar la atención El encargado mapuche articula su significación a partir de la relación entre el pueblo mapuche y el
Estado Chileno, y los procesos de asimilación, a partir de la adaptación del pueblo mapuche a la sociedad chilena:

"porque aqui hay otros problemas que se presentan la asimilación, o la bueno asimilación más que nada que la sociedad dominante está sobre la otras culturas, que muchos mapuches ya están asimilados.

Su narración se elabora a partir de su experiencia de trabajo con políticas emanadas desde el Estado para los pueblos Originarios, primero en relación a la vinculación con la CONADI (Corporación Nacional de Desarrollo Indígena) y luego con el Servicio de Salud Valparaíso San Antonio (SSVSA), particularmente con el Programa Especial de Salud y Pueblos Indígenas (PESPI).

Sobre la relación de la comunidad mapuche con el CESFAM, es posible rescatar el siguiente relato:

"Al Cesfam, al consultorio, para solicitar los permisos, y hacer el trabajo" ... "eee... no el caso de nosotros no, solamente hemos trabajao, el espacio que nos dan"

Se visualiza que dicha relación se refiere a la facilitación del espacio físico por parte del Centro de Salud para la realización de atención de machi, en la cual no se realiza una interacción entre agentes médicos mapuche y occidentales.

Sobre el proceso de atención de machi que gestiona el encargado mapuche, se manifiestan limitantes del trabajo realizado y de las implicancias de la intervención realizada

"eee... yo creo que es más eee, nos falta lo que te había dicho eee un trabajo de eee a posterior, estar, cómo que dó el paciente, o sea, el tratamiento, cómo le fue con el tratamiento, un seguimiento...

Lo anterior debido a que, como se mencionó anteriormente, el trabajo desarrollado por la Asociación se limita a una intervención anual, durante una mañana (de 9 a 14 horas). Limitante que se relaciona con la imposibilidad de realizar un seguimiento del tratamiento administrado por el machi a los usuarios.

\section{Usuaria mapuche}

Sobre el proceso de atención, la usuaria mapuche hace alusión a las modificaciones en la práctica tradicional producto de la relocalización de la práctica médica, volviéndose articuladora su experiencia personal de migración desde la región de la Araucanía a la ciudad de Valparaíso hace diez años:

"parte como lo básico porque de por sí no están en un lugar donde, yo creo que sería otra atención distinta a lo mejor la misma pero cambiaría solamente al estar en su terreno, o sea, supongamos si él atendiera en su casa - están sus cosas es distinta la atención aunque sea la misma tal cual pero hay algo que cambia el ambiente de por sí cambia porque, porque estay atendiendo en $\mathrm{mi}$ casa, no en la casa.."

Considerando lo anterior, la usuaria mapuche refiere sobre la atención de machi en el contexto del CESFAM Rodelillo:

"ya, uno entra ya y le pregunta, supongamos yo tengo voy ya eeehhh, le preguntan a uno qué, cuál es la enfermedad que siente, o sea, no la revisan el médico, solamente con mirarte ellos tienen, o sea por ser mapuche, usted sabe que el machi tiene el poder de saber con mirar a las personas qué es lo que puede tener, algunos le tienen que decirme pas le tienen qur siii, qué medicamento tiene que tomar, depende de lo, del estado de enfermedad que tenga la persona. ."

Aquí se visualiza la forma particular de interacción que tiene el machi con los usuarios, donde coloca una diferencia con la medicina occidental, refiriéndose a que descel a medicina mapuche no es necesario que un diagnóstico y entregar el remedio, sino que basta con la interacción verbal y visual entre usuario y machi. Además otorga al machi la característica de que con la sola mirada puede diagnosticar la enfermedad del usuario, lo cual se relaciona con los poderes sobrenaturales que se le atribuyen al ser machi desde la cultura tradicional mapuche.

\section{Usuaria no mapuche profesional del}

\section{CESFAM Rodelillo}

La usuaria no mapuche profesional del CESFAM Rodelillo, genera como significación articuladora el concepto de respeto a la diversidad étnica que tiene como base la ética profesional. El respeto se refiere a la actitud dispuesta por los sujetos con pertenencia étnica mapuche hacia el proceso de atención en el Centro de Salud desde el modelo biopsicosocial:

"ellos son muy respetuosos, por eso te digo yo, yo lo observo así, yo he trabajado en otros sectores, vienen a su controles, llevan sus remedios, no están criticando las pastillas ni esas cosas, de eso me di, me percaté yo, yo me pongo a observar entonces ellos son respetuosos, pero a nosotros nos cuesta como valorar más toda la parte de medicina de ellos.

Sobre la atención realizada en el CESFAM, agrega que es "muy buena, ellos son muy acogedores", respecto de la actitud con la cual el encargado mapuche y machi se vinculan con los usuarios y profesionales del Centro de Salud, donde visualiza como limitación la intervención esporádica realizada:

"pero podría ser más seguido, como se hace en el sur, que dieran otras cosas, por ejemplo, como las cremas de ruda, en el sur se da, es mucho mas alternativo, es más fuerte el asunto, acá es más débil"
El relato anterior se refiere a la contrastación entre experiencias de atención de machi, que en la región de la Araucanía tienen mayor continuidad y regularidad en el tiempo que en la ciudad de Valparaíso, en la cual se realiza una intervención anual en el Centro de Salud.

\section{Usuario no mapuche}

En el usuario no mapuche las significaciones circulan en torno a la fe, la cual se configura a partir de su cosmovisión y la forma de entender el proceso de sanación, como una limpieza del organismo tanto en un nivel físico, como psicológico:

"ya, tómate estas gotas ya aquí acá y lo lo hacía con fe, lo tomaba y me hacia bien, realmente bien, y lo notaba

Lo anterior, el sujeto lo otorga a partir de su experiencia pasada de adicción con drogas y alcohol, la cual se vuelve un hito importante en su motivación la atención de medicina mapuche.

Sobre el tratamiento que entrega el machi, el sujeto refiere que es a base de yerbas medicinales, manifestando la efectividad de las mismas, enfatizando sobre el hecho de que los remedios entregados provienen de la naturaleza, "que viene de las plantas, todo es en base a plantas...". De ahí su opción y valoración de la medicina mapuche.

Articulando lo narrado por los sujetos, se identifica que aquellos con pertenencia étnica mapuche estructuran como eje articulador de sus significaciones las relaciones que cada uno tiene con la cultur mapuche (tanto a través del conocimiento ancestral como del mantenimiento de prácticas culturales). En cambio los sujetos sin pertenencia étnica mapuche refieren a la relación entre la medicina alternativa y a medicina occidental, ubicando a la primera como predominante, mientras que la medicina mapuche se ubicaría en la categoría de medicinas alternativas, ya que tiene como base elementos provenientes de la naturaleza, como las yerbas medicinales, relacionándose con elementos desde "lo natural", lo cual sería el componente motivador para la atención de medicina mapuche en el CESFAM Rodelillo.

La segunda pregunta de investigación corresponde a, ¿Cómo se articulan los significados otorgados al proceso de atención con la experiencia particula de los sujetos y los procesos de salud intercultural?, la cual plantea una discusión teórica de la temática de estudio.

Si bien en el contexto del CESFAM Rodelillo es posible visualizar ciertas estrategias provenientes desde la política pública para la inclusión de la cultura de usuario en el proceso de atención, orientadas hacia a 
la población mapuche, concretamente de la médica mapuche, estas refieren a estrategias dirigidas a relaciones interetnicas, más que interculturales, puesto que su foco de atención son sujetos con pertenencia étnica. Respecto de estas estrategias, se visualiza un escaso dílogo entre la comunidad mapuche y los profesionales del CESFAM, ya que la coordinación se realiza solo en la interacción entre tres sujetos, el encargado del PESPI, el encargado mapuche de gestionar la atención de machi (que pertenece al Lo Cheuque), y el director del CESFAM, dejando fuera a los demás profesionales del Centro de Salud y los usuarios, tanto mapuches como no mapuches. Fllo se ejemplifica cuando al encargado mapuche se le pregunta sobre la relación de la Asociación con e Centro de Salud, y responde: "eee... no, el caso de nosotros no, solamente hemos trabajao, el espacio que nos dan".

Es el ámbito privado de los sujetos donde es posible visualizar procesos de complementariedad en salud estos últimos referidos a la coexistencia entre conocimientos medicinales distintos:

"Aparte de los medicamentos que son efectivos para ella igual supongamos de repente no sé si tuviera fiebre yo le doy una agüita que de pimpinela que hay en el cerro florece una florcita allá yo le doy de ese y se le quita, y también es efectivo..." (Usuaria mapuche).

La relación que se produce, si tomamos como referente a García Canclini, sería de coexistencia mutua, donde cada uno de ellos tendría su espacio delimitado, la medicina alternativa destinada aquellas enfermedad que son de baja complejidad (dolor de cabeza, resfrío), y la medicina occidental destinada a enfermedad de alta complejidad (intervenciones quirúrgicas). Es en dicho espacio privado que sujetos cultivan plantas medicinales las cuales consumen de forma complementaria a los medicamentos recetados desde el Centro de Salud.

A lo anterior se agregan las diversas dimensiones a las que alude cada sujeto para hacer referencia a los procesos de interculturalidad en salud, lo que también se relaciona con la experiencia de cada sujeto en la construcción de su narración: el encargado mapuche refiriéndose a procesos de relación entre culturas distintas donde históricamente ha habido procesos de dominación sobre la cultura mapuche y esta ha debido asimilarse a la cultura chilena; la usuaria no mapuche identificando la dimensión de la trama de significados que dota de sentido a cultura, y de aquellos elementos que son posibles de modificar o adaptar sin que la cultura ancestral pierda su sentido originario; la usuaria no mapuche profesional del CESFAM Rodelillo, aludiendo a la actitud de respeto de los sujetos que se encuentran en relación con los procesos de interculturalidad; y el usuario no mapuche, que refiere a una dimensión simbólico material de los procesos de interculturalidad relacionados con la cosmovisión o forma de entender el mundo en que los sujetos habitan y se relacionan entre ellos y su entorno.

Tratemos ahora, con los elementos desarrollados, de responder la siguiente pregunta: ise ha cuestionado la política de salud sobre la influencia en la práctica médica mapuche que, en el momento de la atención, el machi y el usuario se encuentren separados por un escritorio o que la duración de la atención sea de quince minutos considerando que ello no es propio de dicha práctica ancestral?

Para dar respuesta a la pregunta planteada no se deben invisibilizar las modificaciones en la práctica médica mapuche ancestral producto de la interacción con la sociedad nacional chilena, más aun considerando que dicha relación está contenida en una política social particular. Es por este motivo que las estrategias emanadas desde el Estado deben repensarse de tanto en tanto para evitar todo tipo de naturalizaciones de los procesos socioculturales de los cuales son producto y productores; es aquí el punto de inflexión que es posible de realizar desde la óptica del Trabajo Social, pensando en el rol desde donde se ha po ejecución de la política pública, pues este en la intervención directa, ha sido desde la ausencia, pues no es un sujeto implicado en el proceso de atención de machi, de allí su no inclusión como sujeto de investigación.

Además, es necesario considerar que el 90\% de quienes se atienden no tiene ascendencia étnica mapuche, mientras que el 10\% sí la posee, lo cual trae consiguo que "...el trabajo es débil, eee como, eеe como de pinzas, como eee bien minucioso porque la población es poca y y hay que identificarlos, hay que identificarlos" (Encargado Mapuche). Las implicancias que tiene dicha relación estadística se relaciona con que los sujetos que practican la medicina mapuche deban adaptar elementos culturales para que el sujeto sin pertenencia étnica pueda comprender el sentido de sanación que ellos desean trasmitir Para hacer alusión a las diferencias entre la práctica médica que se realiza en el CENSFAM Rodelillo y aquella que se realiza en el territorio mapuche se hará alusión al relato de la usuaria mapuche, quien señala sobre la atención en el Centro de Salud:

"Son nosotros mismos, o sea es distinto a ustedes porque ustedes son, como decirle, de pueblo, nosotros somos mapuche... yyy... el o sea él y el machi, el longko y el machi le hicieron así como un ritual a uno en la cabeza, en donde se dice se habló en mapuche pidiéndole a Dios con nuestra lengua... demora como entre cinco o siete minutos, pero yo estuve como quince minutos, o sea, aparte nosotros nos pusimos a conversar otras cosas...

En cambio, en la región de la Araucanía identifica diferencias en los símbolos que acompañan la práctica de sanación y los recursos utilizados por la machi

"La diferencia es que la machi tiene su regüe $e^{2}$ que es su... que es lo sagrado que ella tiene... y ahí puede... ver la orina.. le ve y le dice que es lo que tie puede.. . Sobre la administración del remedio, identifica la siguiente diferencia, en la Araucanía "...no le dan una bolsita de remedios... entonces allá le dan todos los remedios supongamos le dan botellas de dos litros por cada, se toma esto y esto, le dan cuatro litros de remedio y usted se toma esto en la mañana, después de almuerzo y en la tarde y este es solanente ch la i pa que usted descanse. acá no es la bolsita deee remedio, prepárelo así así le dan las indicaciones, todo como uno se lo tiene que preparar...

Queda de manifiesto en el relato anterior la flexibilidad de la cultura mapuche para adaptarse al contexto en el cual se practica, donde se visualizan diferencias respecto de artefactos culturales identitarios diferenciadores en el espacio físico (por ejemplo, la ausencia del regüe en el CESFAM Rodelillo), pero similitudes en la definición de la enfermedad, la cual se mantiene en ambos contextos. Queda como desafío de la política pública entonces incorporar dichos elementos culturales en la configuración de un espacio físico que posibilite la reproducción ancestral de la práctica médica mapuche

\section{Reflexiones: el aporte desde el}

\section{Trabajo Socia}

A partir de los elementos mencionados en los apartados anteriores, es necesario realizar una reflexión respecto del marco sociopolítico en el cual se contextualiza la atención de machi en el CESFAM Rodelillo, lo cual vendría siendo el aporte particular realizado desde el Trabajo Social, como disciplina y profesión abocada a la deconstrucción y desnaturalización de los procesos sociales, teniendo como consideración e imperativo ético la subjetividad heroica (De la Aldea \& Lewkowics, 2004), respecto del rol de facilitador de procesos en la intervención social.
Sobre investigaciones en salud intercultural

Las investigaciones en esta temática han dejado de manifiesto la importancia que cobra la identidad étnica en los procesos de interculturalidad en salud respecto de la pertinencia de las iniciativas de integración de medicina mapuche en Centros de Salud (Álvarez, 2005), sobre todo respecto de lo que sucede en el contexto urbano, donde ocurre una resignificación de la identidad étnica mapuche, la cual está marcada por la relocalización de las prácticas médicas y por los procesos de adaptación de dichas prácticas a un contexto regulado y normativo, como lo es el de los Centros de Salud.

Como contenedor de los procesos de interculturalidad tenemos lo que García Canclini (1997) refiere como hibridación sociocultural, la cual "no es una simple mezcla de estructuras prácticas sociales discretas, puras, que existían en forma separada, y a combinarse, generan nuevas estructuras y nuevas práccicas. A veces esto ocurre de modo no planeado, o es el te Can Canclini, p. 112). Es en las metrópolis donde se observan mezclas multiculturales, la heterogeneidad es multitemporal, la industria no elimina las artesanías, la democratización no suprime los hábitos autoritarios, ni la cultura escrita las formas antiguas de comunicación oral, lo cual puede ser resultado del acceso desigual a los bienes de la modernidad. Las experiencias de hibridación son parte de los conflictos de la modernidad latinoamericana, es en estos contextos donde se (re)producen las prácticas de integración de la medicina mapuche en centros de salud, es en dichas experiencias donde se visualiza la coexistencia de elementos tradicionales, como la práctica médica mapuche y sus ritos ancestrales con elementos propios de la cultura occidental moderna, aquello relacionado con el desarrollo tec nológico y en tratamiento de la enfermedad, lo cua es posible de visualizar en el CESFAM Rodelillo.

Si nos preguntamos, ise cristalizará el sistema médico mapuche al incluirse en los Centros de Salud de atención primaria?, una respuesta que se puede entregar es que no, puesto que los sistema médicos, como sistemas culturales, al ser tramas de significación, son dinámicos, y por tanto pueden modificarse al entrar en interrelación con otras tramas de significado; pero sí es posible identificar elementos que otorgan sentido a dichos sistemas culturales y que por tanto no son modificables para que dicho sistema pierda sentido para los sujetos 
que de él participan, estos son los que se relacionan directamente con la cultura particular de la cual es parte el sistema médico.

Lo anterior queda de manifiesto en el relato de la usuaria mapuche, quien contrasta lo que ocurre en la región de la Araucanía con lo que sucede en el CESFAM Rodelillo, respecto de la atención de machi (haciendo alusión al acá de la ciudad de Valparaíso y al allá de la región de la Araucanía) particularmente de la administración de la yerba medicinal: "...Allá le dan todos los remedios supongamos le dan botellas de dos litros por cada, se toma esto y esto"

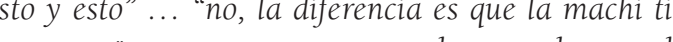
ne su regue, que es su... que es lo sagrado que ell tiene... (en la Araucania). En cambio el remedio en el CESFAM Rodelillo se entrega en una bols sellada. Otra diferencia sería que la atención en la región de la Araucanía se realizaría en casa de la machi, en cambio en Valparaíso se realiza en una sala de atención del Centro de Salud.

Un elemento que tenemos que tener en cuenta para responder la pregunta planteada es lo señalado por Castañeda \& Pantoja (2004), quienes desde un mirada sociohistórica señalan la flexibilidad de la cultura mapuche al entrar en relación, primero con la cultura española, y luego con la cultura nacional chilena, lo cual ha permitido su persistencia en tiempo a pesar de los diversos confictos de relación Las investigaciones relacionadas con la interculturalidad en salud deben tener presente que $l$ intercultural no solo debe ser entendido en término de realizar una intervención desde un modelo de salud indígena (en este caso, atención de machi), pues es reducir dichos procesos socioculturales a una mínima expresión; la interculturalidad en salud debe ser entendida como procesos de interacción entre sujetos que tienen prácticas culturales distinas (y por ende, visiones de mundo diversas), pues si no, estaríamos hablando de salud interétnica y no de salud intercultural. Es aquí donde causa ruido el relato del Usuario no Mapuche, cuando alude a la complementariedad ideal de ambas medicinas:

"Deberian funcionar más entre los dos medicinas, o sea, saber lo que sabes tú en los libros y lo que sé en las plantas y... apoyarse más $y$ ver eso poh, por ejemplo ya llegai a la asistencia pública y llegai con con el brazo dislocao ya se le salió el codo, y quedai con el brazo pa afuera... para qué hacer tajo y agarrar el hueso si por fuera puede hacerse, aguantar el dolor no ma yyy com-

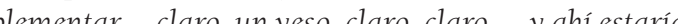
plen los dos por ejemplo, teni un ejemplo de se juntarian las dos medicinas, uno hace el trabajo eee poniendo el hues en su lugar y después viene la parte yeso que lo pondría el hospital.

Debemos tener presente lo que nos señala García Canclini al advertir la reconceptualización de la noción de cultura, desde el aspecto teórico-metodológico, de no entender esta como un paquete de rasgos que identifica a una sociedad de otra, sino que concebir lo intercultural, o como refiere el autor, "lo cultural", "como sistema de relaciones de sentido que identifica "diferencias, contrastes y comparaciones» (Appadurai, 1996: 12-13), el "vehículo o medio por el que la relación entre los grupos es llevada a cabor

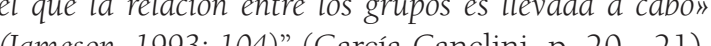
Jameson, 1993: 104)" (Garcia Canclini, p. 20 - 21). Este autor nos invita a complejizar los estudios socioculturales y a prestar atención a las mezclas culturales que generan relación entre los diversos grupos, donde es necesario, para entender dichos grupos, describir cómo se apropian y reinterpretan las formas culturales adquiridas tanto en un nivel simbólico como material.

\section{Sobre la Política de Salud y Pueblos Indígenas} en Chile

Si pensamos en el proceso de atención que se realiza en el CESFAM Rodelillo, en el cual se incluyen las diferentes atenciones desde el enfoque biopsicosocial, la intervención realizada por la Asociación Indígena Rayen Foye, teniendo en cuenta que esta sería una estrategia de salud intercultural, sería una anécdota en la vida cotidiana del Centro de Salud. Si bien la Real Academia Española de la Lengua (RAE) define como anécdota un "suceso curioso y poco conocido, que se cuenta en dicho relato", o como "suceso circunstancial o irrelevante", aquí se resignifica dicho concepto, y se utiliza para interpelar a la Política de Salud y Pueblos Indígenas, para la diversificación de las estrategias de inclusión de la cultura del usuario al proceso de atención, la cual se limita a la difusión informativa de la atención y luego de la atención, como lo relata el encargado mapuche:

"O pre trabajo, pre atención, son como tres o dos semanas, y hago ese trabajo, con otras personas, entrego información, hecha, impresa, de qué se trata la atención, el día, de qué se trata, qué tratamientos puede hacerse... luego se hace la atención ese día..."

El concepto de anécdota se utiliza para visualizar el proceso de atención de medicina mapuche dentro del proceso total de atención del CESFAM Rodelillo, desde el enfoque biopsicosocial, el cual es más amplio que la intervención que realiza la comunidad mapuche. No se considera un suceso irrelevante, por lo mismo que se considera como un fenómeno de investigación, el cual es significado por los sujetos implicados de este. No se utiliza en forma peyorativa respecto de la intervención de la comunidad mapuche, sino que más bien como condición de posibilidad para intensificar y diversificar dicho trabajo realizado con el apoyo del Servicio de Salud Valparaíso San Antonio.

Se observa la adquisición de elementos culturales en el sistema médico mapuche que se modifican y adaptan a las normativas del modelo biopsicosocial de salud que desarrolla el CESFAM Rodelillo (la forma en que se entrega el remedio, la distribución espacial entre machi, longko y usuario), apreciándose una mapuche mapuche al sistema médico occidental, construida históricamente. Al parecer, las estrategias de inclusión de la medicina mapuche en el CESFAM no han tenido un impacto significativo en el quehacer de los profesionales del Centro de Salud debido a que estos no participan en la gestión de la intervención desarrollada. La atención de salud por agentes mapuche debiera contar con un mayor número de interve de intervenciones o que permitiria que dichas estrategias de interculturalidad en salud dejaran de ser una anécdota en el proceso de atención del CESFAM Rodelillo y pasaran a formar parte de la atención regular que ofrece el CESFAM en pos de generar procesos de complementariedad entre sistemas médicos.

Se debe agregar que si nos referimos a relaciones interétnicas, como lo hace la Política de Salud y Pueblos Indígenas, habría que preguntarse si el espacio que se le concede a la medicina mapuche para su actuación en el contexto de Centro de Salud es el adecuado, pues considerando la cosmovisión de este pueblo originario, lo más pertinente para el desarrollo de su sistema médico es contar con un espacio particular, como una ruka ${ }^{3}$, para realizar la atención y no una sala del CESFAM como se hace actualmente, lo cual tiene como consecuencia la asimilación del sistema médico mapuche a los procedimientos del sistema médico occidental, puesto que, como se aprecia especialmente en la narración de la usuaria mapuche, el espacio físico y la distribución espacial (y por tanto las significaciones simbólico materiales hacia este), se relacionan con la forma en la cual configuran el mundo los sujetos con pertenencia étnica mapuche.

Si la política de salud tiene un enfoque de salud intercultural, surge la siguiente pregunta: ¿Cuáles

3 Vivienda tradicional mapuche.

4 Articulo $2^{\circ}$, Decreto $\mathrm{N}^{\circ} 42$ de 2004, del Ministerio de Salud, Reglamento para el Ejercicio de las Profesiones Auxiliares de la Salud y de los Recintos en que Estas Se Realizan. son las estrategias de integración de la medicina popular chilena en los Centros de Salud?, si bien se entiende la medicina popular chilena como "la medicina popular tradicional chilena, entendida como as actividades y procedimientos de recuperar y mantener la salud, de origen sociocultural autóctono en el país, ejercida por sanadores formados tradicionalmente en sus propias comunidades de pertenencia y que gozan del respeto de estas, quedará al margen de la aplicación de este reglamento", al parecer estas actividades no han ocupado los espacios de los centros de salud; queda el desafío de diversificar las estrategia para integrar este tipo de medicina al espacio de los centros de salud, puesto que dichas prácticas tradicionales son cotidianas en la vida de los sujetos, por ejemplo "quebrar el empacho", "sacar aires", incluso la ingesta de yerbas medicinales (estas últila práctica médica de los pueblos originarios con la de los conquistadores)

Es posible aventurar que más que un enfoque de interculturalidad, la política tendría un enfoque multicultural, recordando la diferencia entre ambos conceptos planteada en la introducción haciendo alusión a lo señalado por García Canclini, puesto que se acepta la diversidad cultural pero las estrategias estarían dirigidas hacia la adaptación de lo heterogéneo, en este caso el pueblo mapuche al sistema médico oficial. Esto se relaciona con el relato del encargado mapuche de gestionar la atención de machi: "Para que ellos sepan que existe un modelo de salud indigena para ellos, entonces ellos tienen que eee, digamos, aceptar ese modelo finalmente... ahora si no lo aceptan ya no es problema de cada uno..."

Es aquí donde se visualizan los impactos de la Política de Salud y Pueblos Indígenas en los sujetos, en especial aquellos con pertenencia étnica mapuche vinculados en la gestión de la intervención desde la medicina mapuche, y de la relación entre la cultura hegemónica y las culturas subordinadas, la cual, en términos de Gramsci (en: Alvarez N, 2005), habría sedimentado en el sentido común de los sujetos la ideología dominante, en la cual el modelo biopsicosocial desarrollado por la medicina oficial es el predominante y el ordenador de la práctica médica. Quizás sea necesario recordar las estrategias de salud intercultural planteadas por Alarcón, Vidal, \& Neira (2003) para desarrollar estrategias efectivas de diálogo entre culturas diferentes y sujetos con mas son producto de los procesos de hibridación de 
mallas de significación particulares. Estas consisten en la interaccion médica de saberes distintos (lo cual requiere de transformaciones estructurales) de la comunicación intercultural, además del desarrollo de procesos de validación y negociación cultural entre usuarios y profesionales.

La invitación es finalmente a repensar la política pública de salud en general, y las acciones orientadas los pueblos indígenas y al resto de la sociedad en particular; a diversificar las estrategias de integración de la cultura del usuario en el proceso de atención tanto respecto de la relación interétnica (referida a sujetos pertenecie a relacio le signifcados constuida de forna paricular de car uno de los usurios), sobre todo conticular de cada uno de los usuarios), sobre todo considerando las características propias de los espacios territoriale y de los microcontextos sociales en los cuales los sujetos entran en relación directa, puesto que se visualiza la falta de una política, que dentro de la concepción de intercultura acionados con las formas en que los sujetos llevan cabo sus prácticas culturales, las cuales serán diversas a partir de la malla de significación particular que cada uno construya.

\section{Conclusiones}

El aporte desde el Trabajo Social con esta investigación es el dejar de pensar la salud desde el etnocentrismo, no son las llamadas medicinas alternativas las que complementarían la medicina oficia (modelo biopsicosocial y biomédico), y por ende estas se encontrarían en un segundo o tercer plano, sino que debe ser incluso un imperativo ético el dilogo efectivo que permita el enriquecimiento muto, no es "entregar un espacio para que la otra medicin desarrolle sus prácticas" (como facilitar una sala de atención para que el o la machi desarrolle su prác tica ancestral), sino que realizar un intercambio de conocimientos, técnicas y formas de entender el mundo en el cual los sujetos se sitúan (aludiendo la complementariedad que narra el usuario no mapuche).

Los sujetos implicados en la atención de machi en el CESFAM Rodelillo significan su experiencia a parti de la visión de mundo que construyen en relación a la salud-enfermedad-sanación, concordando en las limitaciones de realizar una intervención anual en dicho espacio. Aluden de forma implícita a la contextos donde se reproduce su practica médica ancestral, identificando modificaciones relaciona-
das con elementos materiales y simbólicos, como la distribución del espacio físico y la administración del remedio, pero la mantención del sentido de espiritualidad y relación con el medio natural que contiene la medicina mapuche.

Queda abierta la pregunta ¿Qué es lo intercultural de la interculturalidad en salud?, o más bien, ¿Qué tiene de intercultural la Política de Salud y Pueblos Indígenas? Si entendemos la interculturalidad desde la dimensión de estrategia estatal de inclusión de la cultura del usuario en el proceso de atención, nos daremos cuenta de que dichas estrategias se limitan a conceder un espacio de autonomía relativa a los sistemas méclcos de los pueblos originarios (donde estos deben adaptarse al modelo hegemónico), y de regulación de las medicinas alternativas y complementarias que deben cumplir un cierto estándar y adecuación al modelo biopsicosocial. Por lo tanto, su enfoque sería interétnico, referido a la relación entre la cultura nacional chilena y la cultura mapuche. Ahora bien, si entendemos lo intercultural desde la dimensión de proceso sociocultural, lo cual es de por sí más amplio, nos daremos cuenta de que, por ser sujetos sociales, en nuestras vidas cotidianas nos encontramos insertos en procesos interculturales con sujetos que construyen mallas de significaciones particulares (ya sea de negociación, relación, intercambio, préstamos recíprocos, conflictos, etc.), y es precisamente en estos procesos donden debe hacer énfasis las acciones estatales para integrar las diversas formas culturales de los sujetos a los cuales van dirigidas dichas estrategias de salud.

\section{Referencias}

AlarCón, A. M., VIDAL, A., \& NEIRA, J. (2003). Salud Intercultural: Elementos para la construcción de sus bases conceptuales. Revista Medica de Chile, php?

ONSO, L. E. (2003). La Mirada Cualitativa en Sociología: Una Aproximación Interpretativa (Segunda Edición ed.). Madrid, España: Fundamentos

ÁLVAREZ, N. (2005). Interculturalidad en salud y desarro$1 l o$ de comunidades mapuche: Estudio de Caso en Makewe Pelale. Tesis presentada como parte de los requisitos para optar al Grado de Magister en Gestion en Desa- Rural y Agricultura Sustentable, Unversidad Católica de Temuco, Temuco. En: http///biblioteca uct. cl/tesis/nancy-alvarez/tesis.pdf

ARRIARÁN, S. (2000). La Hermenéutica de Clifford Geertz. Iztapalapa. Revista de Ciencias Sociales y $\mathrm{Hu}-$ manidades (49), 95 - 106. En. http.//48.206.53.230/ revistasuam/iztapalapa/include/getdoc. php?id=743\&article $=757 \&$ mode $=$ pd
BRUNER, J. (1998). Actos de significado. Más allá de la revolución cognitiva. Madrid, España: Alianza Editorial.

CASTAÑEDA, P., \& PANTOJA, M. Á. (2004). La Gente de la Tierra... En la Tierra de Otra Gente: Migración Mapuche Contemperánea Hacia la Región de Valparaíso. Cuadernos Interculturales - Universidad de Valparaiso, 2 (002), 7 - 17. En: http://redalyc.uaemex. $\mathrm{mx} / \mathrm{pdf} / 552 / 55220202$.pdf

DE LA ALDEA, E., \& LEWKOWICS, I. (2004). La Subjetividad Heróica: Un obstáculo en las prácticas comunitarias en la salud. Buenos Aires: Mimeo.

GADAMER, H. -G. (1998). Verdad y Método II. Salamanca: Ediciones Sígueme.

GARCÍA CANCLINI, N. (1997). Culturas Híbridas y Estrategias Comunicacionales. Estudios sobre las Culturas 128. En hap//ralyc pdf

GARCIA CANCLINI, N. (2004). Diferentes, Desiguales y Desconectados. Mapas de la interculturalidad (1 ${ }^{\mathrm{a}}$ Edición ed.). Barcelona, España: Gedisa.

GEERTZ, C. (1994). Conocimiento Local (Primera Edición ed.). (A. L. Bargados, Trad.) Barcelona, España: Ediciones Paidos.

GEERTZ, C. (2003). La interpretación de las Culturas. Barcelona, España: Gedisa.

GEERTZ, C. (1996). Usos de la Diversidad (Primera Edición ed.). (M. J. (3), Trad.) Barcelona, España: Ediciones Paidós.

LOF CHEUQUE; Revista mapuche urbana: "Warriache Lafquen"; 9 Edición Anual 2011 - 2012

MINSAL. (2003). Politica de Salud y Pueblos Indigenas. Santiago.

MINSAL. (2011). Programa Especial de Salud y Pueblos Indigenas. Subsecretaría de Redes Asistenciales - División de Atención Primaria.

NAVARRO, P., \& DíAZ, C. (1994). Análisis de Contenido. En J. M. Delgado, \& J. Gutierrez, Métodos y Técnicas cualitativas de Investigación en Ciencias Sociales (págs. 177 - 124): Madrid, España Sintesis:

STRAUSS, A., \& CORBIN, J. (2002). Bases de la Investigacion Cualitativa. Tecnicas y Procedimientos para (a) Medellin. Editoria Universidad de Antioquia.

TAYLOR, S. J., \& BOGDAN, R. (1987). Introducción a los Metodos Cualitativos de Investigación. La Búsqueda de Ediciones Paidós Ibérica, S. A.

VIDAL, A; EN: CITARELLA, L. (1995). Medicinas y Culturas en la Araucanía. Santiago, Chile: Editorial Sud- 